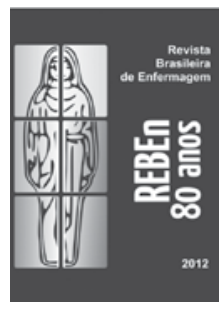

\title{
Enfermeiros dos grandes hospitais públicos no Rio de Janeiro: características sociodemográficas e relacionadas ao trabalho
}

Nurses of large public hospitals in Rio de Janeiro: socio demographic and work related characteristics Enfermeros de los grandes hospitales públicos de Rio de Janeiro: características socio-demográficas y laborales

\author{
Rosane Härter Griep', Maria de Jesus Mendes da Fonseca", Enirtes Caetano Prates Melo", \\ Luciana Fernandes Portela"', Lucia Rotenberg'"I \\ ' Fundação Oswaldo Cruz, Instituto Oswaldo Cruz, Departamento de Biologia. Rio de Janeiro-RJ, Brasil. \\ "Fundação Oswaldo Cruz, Escola Nacional de Saúde Pública, \\ Departamento de Epidemiologia e Métodos Quantitativos em Saúde. Rio de Janeiro-RJ, Brasil. \\ I'I Fundação Oswaldo Cruz, Instituto Oswaldo Cruz, Laboratório de Educação em Ambiente e Saúde. Rio de Janeiro-RJ, Brasil.
}

Submissão: 28-02-2013 Aprovação: 22-05-2013

\section{RESUMO}

O objetivo do estudo foi analisar características sociodemográficas e de trabalho de enfermeiros que atuam em hospitais públicos. Realizou-se estudo epidemiológico, seccionais, envolvendo 3.229 enfermeiros dos dezoito maiores hospitais públicos no município do Rio de Janeiro. Observou-se predominância feminina $(87,3 \%)$ e idade média de $39,9 \pm 10$ anos. Cerca de $7 \%$ referiram ter titulo de mestrado e/ou doutorado, 58,5\% formaram-se em instituições públicas e 24,5\% trabalhavam no setor saúde antes de serem enfermeiros. Metade pensou em abandonar a Enfermagem e quase um quarto se considera insatisfeito com a profissão. Cerca de $10 \%$ esteve procurando emprego fora e 30\% na própria Enfermagem. Entre os homens foi mais frequente o trabalho noturno, mais de um emprego e carga semanal de trabalho mais elevada. O estudo apontou aspectos desafiadores para os enfermeiros/as. Em função de sua abrangência, os resultados podem subsidiar estratégias de melhoria das condições de trabalho nos hospitais públicos. Descritores: Enfermeiros; Condições de Trabalho; Satisfação no Emprego; Força de Trabalho, Jornada de Trabalho; Saúde do Trabalhador.

\begin{abstract}
The study aimed at analyzing socio-demographic and working characteristics of nurses from public hospitals. It was carried out a cross-sectional study, involving 3.229 nurses from the eighteen largest public hospitals of the city of Rio de Janeiro. It was observed a feminine predominance $(87.3 \%$ ), with mean age of $39.9 \pm 10$ years. Around $7 \%$ referred having master or doctorate degree, $58.5 \%$ got their degree from public institutions and $24.5 \%$ used to work at the health sector before becoming nurses. Half the group has thought of abandoning their career, and almost a quarter is unsatisfied with their profession. Around $10 \%$ searched for a job outside nursing area in the previous month and 30\% searched for a job in the same working area. Night work, engagement in more than one job and long professional work hours were more frequently found among men. The study has pointed challengeable aspects of nurses' profession. Results can subsidize support strategies to improve the working conditions in public hospitals due to their comprehensiveness.
\end{abstract}

Key words: Nurses; Working Conditions; Job Satisfaction; Labor Force; Work Hours, Occupational Health.

\section{RESUMEN}

El objetivo fue analizar características socio demográficas y de trabajo de enfermeros que trabajan en hospitales públicos. Fue realizado un estudio epidemiológico transversal incluyendo 3.229 enfermeros, de los 18 hospitales mayores públicos de la ciudad de Río de Janeiro. Se observó predominio del sexo femenino $(87,3 \%)$ y media de edad de $39,9 \pm 10$ años. Aproximadamente $7 \%$ reportó tener maestría y/o doctorado, 58,5\% fue formado en instituciones públicas y $24,5 \%$ trabajaba en cuidado de salud antes de ser enfermero. La mitad pensó en dejar la profesión y casi una cuarta parte están insatisfechos con el trabajo. Aproximadamente 10\% estaba buscando trabajo fuera de la enfermería y 30\% buscó empleo en la enfermería. Los hombres informaron con mayor frecuencia el trabajo nocturno, más de un empleo, además de jornada de trabajo profesional más alta. El estudio demostró aspectos desafiantes para los enfermeros. Por su magnitud los resultados pueden apoyar las estrategias para mejorar las condiciones de trabajo en hospitales públicos.

Palabras clave: Enfermeros; Condiciones de Trabajo; Satisfacción en el Trabajo; Fuerza de Trabajo; Horas de Trabajo; Salud Laboral

AUTOR CORRESPONDENTE Rosane Härter Griep E-mail: rohgriep@gmail.com 


\section{INTRODUÇÃO}

Hospitais gerais são organizações de alta complexidade que integram o setor de serviços da sociedade contemporânea. O processo de trabalho nestas instituições inclui especialidades pouco articuladas, hierarquia entre os grupos profissionais envolvidos no cuidado e discurso médico hospitalar dominante ${ }^{(1)}$. Nesses cenários, agregam-se tanto o componente técnico quanto as (inter)relações que se estabelecem entre os atores responsáveis pelo ato de cuidar ${ }^{(2)}$.

Nos hospitais públicos, o trabalho do enfermeiro envolve o gerenciamento da assistência de enfermagem, visando a eficiência e humanização do cuidado $^{(3)}$. O enfermeiro deve planejar a assistência e coordenar a equipe de enfermagem além de prestar cuidado direto ao paciente assumindo procedimentos de maior complexidade e prescrevendo cuidados. Portanto, desenvolve de forma compartilhada atividades assistenciais e gerenciais, que constituem as duas principais dimensões do processo de trabalho ${ }^{(3-4)}$, o que frequentemente implica equacionar conflitos, lidar com insatisfações e manter o poder disciplinar da equipe ${ }^{(5)}$.

Além disso, é reconhecido que as características de trabalho da Enfermagem envolvem as longas jornadas e o trabalho intensivo, plantões noturnos e nos fins de semana, multiplicidade de funções, a repetitividade e a monotonia, a intensidade e o ritmo excessivo de trabalho, o elevado esforço físico e posições incômodas ${ }^{(3,5)}$. Cabe também destacar os múltiplos vínculos empregatícios, prática comum entre os profissionais da saúde no Brasil $^{(6)}$. A interação complexa entre esses fatores tem sido relacionada aos danos à saúde do trabalhador, tais como sintomas musculoesqueléticos ${ }^{(5)}$, sofrimento psíquico, estresse psicossocial do trabalho ${ }^{(6)}$ e acidentes $^{(7)}$, o que contribui para o absenteísmo ${ }^{(8)}$ e que acrescenta problemas à qualidade da assistência ${ }^{(9)}$.

Pouco se conhece sobre as características específicas do enfermeiro no contexto das categorias profissionais da Enfermagem e nenhum estudo abrangeu amostra ampla o suficiente que permitisse descrever as relações entre a saúde e o trabalho dos enfermeiros nos hospitais próprios do Sistema Único de Saúde (SUS). Esse foi o tema central do Estudo da Saúde dos Enfermeiros dos grandes hospitais públicos no Rio de Janeiro. O presente artigo tem como objetivo descrever aspectos metodológicos do referido estudo e apresentar características sócio-demográficas e de trabalho dos enfermeiros e enfermeiras que atuam nesses hospitais.

\section{MÉTODOS}

A investigação "Trabalho noturno e sua associação com fatores de risco para doenças cardiovasculares entre enfermeiros" foi divulgada nos hospitais como o "Estudo da Saúde dos Enfermeiros". Utilizou metodologia seccional, com o objetivo principal de avaliar a associação entre características do trabaIho e a saúde do conjunto dos enfermeiros e enfermeiras, que prestam assistência em grandes hospitais públicos do município do Rio de Janeiro. Para a obtenção da população de estudo foram selecionados no Cadastro Nacional de Estabelecimentos de Saúde (CNES) todos os hospitais públicos com mais de 150 leitos, distribuídos em todas as Regiões Administrativas do município. A partir desse cadastro, 19 hospitais foram considerados elegíveis: quatro hospitais municipais, cinco estaduais, cinco federais ligados ao Ministério da Saúde, três hospitais universitários (um deles de uma universidade estadual) e dois institutos ligados ao Ministério de Ciência e Tecnologia. Um dos hospitais estaduais sofreu um incêndio na época da coleta dos dados e por isso foi desativado. A população de trabalhadores foi, então, transferida para outro hospital estadual que se incorporou ao estudo. Com isso, os trabalhadores enfermeiros de 18 hospitais foram convidados a participar da investigação. Todos os hospitais foram visitados e reuniões foram agendadas com as chefias de enfermagem como forma de divulgação da metodologia e propósitos do estudo. A partir da autorização da direção de cada hospital, obteve-se a listagem dos nomes e setores de trabalho dos enfermeiros ali lotados. Foram considerados não elegíveis: os licenciados, os substituídos por outros profissionais (por acordo pessoal) através de pagamento e os cedidos ou exonerados. Do total de 3.904 elegíveis, $3229(82,7 \%)$ devolveram questionários preenchidos. As perdas estão relacionadas a recusas $(n=478)$, àqueles não encontrados em mais de três visitas ao setor durante dois meses $(n=128)$ e a férias $(n=69)$, totalizando $675(17,3 \%)$.

Cada enfermeiro foi procurado pela equipe de entrevistadores treinados, na maioria enfermeiras, para a abordagem pessoal e explicação dos objetivos do estudo e convite à participação. Após a assinatura do Termo de Consentimento Livre e Esclarecido, o enfermeiro recebia o questionário cuja devolução era feita pessoalmente (em dias previamente agendados) ou por meio de urnas disponibilizadas nos hospitais. O período de coleta dos dados ocorreu de Março de 2010 a Novembro de 2011.

\section{Instrumento de coleta dos dados}

O questionário, autopreenchível, continha perguntas cuidadosamente selecionadas a partir de estudos prévios sobre o tema. Todas as escalas internacionais incluídas tinham descrição do processo da adaptação para o português brasileiro, além de estimativas de confiabilidade e validade demonstradas. A versão final continha três grandes blocos de perguntas: I - Características do trabalho profissional e doméstico: trabalho anterior à função de enfermeiro, número e tipo de vínculos profissionais, carga de trabalho semanal, tempo de trabalho na ocupação; estresse psicossocial do trabalho, satisfação com a profissão; turno de trabalho, possibilidade de dormir ou repousar durante o trabalho noturno; número de horas dedicadas ao trabalho doméstico e sobrecarga de trabalho doméstico; II - Características relacionadas à saúde: doenças crônicas diagnosticadas por médicos, distúrbios do sono, distúrbios psíquicos menores, índice de massa corporal (obtido por meio do peso e altura referidos), ganho de peso desde os 20 anos de idade, atividades físicas de lazer, tabagismo, uso de bebida alcoólica, consumo de alimentos fritos, frutas e verduras e III - Características de posição socioeconômica: idade, escolaridade, situação conjugal, renda familiar, número de dependentes e tipo de universidade (pública ou privada) em que cursou a graduação.

O questionário foi submetido a cinco etapas de pré-testes nas quais a ordenação dos itens foi aprimorada, bem como a avaliação da compreensão das perguntas. 


\section{Tratamento estatístico dos dados}

As informações extraídas do questionário foram inseridas em banco de dados utilizando-se o software Access (Microsoft Corporation, versão 2010) após revisão e codificação por revisores treinados. A detalhada revisão e "limpeza" do banco de dados identificou e corrigiu erros de consistência por falha de codificação, digitação ou inconsistência entre respostas. No presente artigo foram descritas variáveis selecionadas de características sociodemográficas e relacionadas ao trabalho profissional. A análise dos dados foi feita no software SPSS (Statistical Package for the Social Sciences, IBM, versão 16), utilizando-se estatísticas descritivas por meio de análises univariadas e bivariadas. Foram empregados os testes qui-quadrado de Pearson para variáveis categóricas e ANOVA para variáveis contínuas, considerando-se nível de significância de 5\% $(p<0,05)$.

\section{Considerações éticas e retorno dos dados aos participantes}

O projeto foi aprovado pelo Comitê de Ética em pesquisa da Fiocruz sob n 472/08. Os questionários eram lacrados pelos participantes após o preenchimento e sua identificação era feita por meio de código numérico. Os resultados de cada hospital do estudo foram resumidos em folhetos explicativos e entregues a todos os enfermeiros dos hospitais (independentemente da sua participação). Foram realizadas palestras para a discussão dos resultados identificados em cada hospital do estudo.

\section{RESUL TADOS}

A adesão por natureza jurídica do hospital variou entre $68,4 \%$ (hospitais municipais) e 92,5\% (hospitais universitários), sendo que $82,7 \%$ do total de elegíveis responderam ao questionário.

O grupo era predominantemente feminino $(87,3 \%)$ e a idade média dos trabalhadores foi de 39,9 10 anos. A maioria era casada, tinha concluído cursos de especialização lato sensu, referiu renda per capita superior a $\mathrm{R} \$ 1.000,00$ e cor de pele branca. Comparados às mulheres, os enfermeiros eram mais velhos e mais frequentemente casados/vivendo em união. Mais da metade dos entrevistados informou cor de pele branca (tabela 1).

Observou-se parcela pequena de enfermeiros/as que referiram ter titulo de mestrado e/ou doutorado. Comparados às mulheres, os homens referiram mais frequentemente ter graduação e mestrado. Entre as mulheres foi mais frequente títulos de especialização e doutorado $(p=0,016)$. A proporção de enfermeiros/as com mestrado ou doutorado variou de $3 \%$ nos hospitais municipais e estaduais a 15\% nos hospitais universitários (dados não mostrados na tabela). Pouco mais da metade dos trabalhadores/as formou-se em instituições públicas, sendo entre as mulheres a frequência um pouco mais elevada do que entre os homens (tabela 1).

Tabela 1 - Características sociodemográficas dos enfermeiros, segundo o sexo. Grandes hospitais públicos do Município do Rio de Janeiro/RJ, 2010-2011.

\begin{tabular}{|c|c|c|c|c|c|c|c|}
\hline \multirow[t]{2}{*}{ Características } & \multicolumn{2}{|c|}{$\begin{array}{l}\text { Mulheres } \\
(n=2818)\end{array}$} & \multicolumn{2}{|c|}{$\begin{array}{l}\text { Homens } \\
(n=411)\end{array}$} & \multicolumn{2}{|c|}{$\begin{array}{c}\text { Total } \\
(n=3229)\end{array}$} & \multirow[t]{2}{*}{$\begin{array}{l}\text { Valor } \\
\text { de } p\end{array}$} \\
\hline & $\mathrm{N}$ & $\%$ & $\mathrm{n}$ & $\%$ & $\mathrm{n}$ & $\%$ & \\
\hline \multicolumn{8}{|l|}{ Faixas etárias (anos) } \\
\hline 22 a 35 & 1105 & 39,7 & 145 & 35,9 & 1250 & 39,2 & $0,052 * *$ \\
\hline 36 a 45 & 793 & 28,5 & 111 & 27,5 & 904 & 28,4 & \\
\hline 46 a 68 & 883 & 31,8 & 148 & 36,6 & 1031 & 32,4 & \\
\hline \multicolumn{8}{|l|}{ Situação Conjugal } \\
\hline Solteiros/ & 688 & 24,7 & 76 & 18,8 & 764 & 23,9 & $<0,001 *$ \\
\hline Casados/em união & 1557 & 55,9 & 276 & 68,3 & 1833 & 57,5 & \\
\hline Separados/viúvos & 541 & 19,4 & 52 & 12,9 & 593 & 18,6 & \\
\hline \multicolumn{8}{|l|}{ Grau de instrução } \\
\hline Doutorado & 20 & 0,7 & 1 & 0,2 & 21 & 0,7 & $0,016^{*}$ \\
\hline Mestrado & 178 & 6,4 & 32 & 7,9 & 240 & 6,6 & \\
\hline Especialização & 1916 & 68,7 & 249 & 61,6 & 2165 & 67,8 & \\
\hline Superior & 673 & 24,1 & 122 & 30,2 & 795 & 24,9 & \\
\hline \multicolumn{8}{|l|}{ Instituição de graduação } \\
\hline Pública & 1662 & 59,6 & 205 & 50,7 & 1867 & 58,5 & $0,001^{*}$ \\
\hline Privada & 1125 & 40,4 & 199 & 49,3 & 1324 & 41,5 & \\
\hline \multicolumn{8}{|l|}{ Renda per capita (em reais) } \\
\hline$<1000,00$ & 373 & 14,2 & 64 & 17,3 & 434 & 14,6 & $0,248 * *$ \\
\hline De 1000,00 a 2000,00 & 1123 & 43,1 & 160 & 43,1 & 1283 & 43,1 & \\
\hline$>2000,00$ & 1112 & 42,7 & 147 & 39,6 & 1259 & 42,3 & \\
\hline \multicolumn{8}{|l|}{ Cor de pele autorreferida } \\
\hline Branca & 1542 & 57,3 & 233 & 59,9 & 1775 & 57,7 & $0,024 *$ \\
\hline Preta & 319 & 11,9 & 28 & 7,2 & 347 & 11,3 & \\
\hline Parda & 828 & 30,8 & 128 & 32,9 & 945 & 30,7 & \\
\hline
\end{tabular}

*teste de qui-quadrado de Pearson; ** teste de tendência linear excluiu-se ignorados. 
Entre os homens foi mais frequente o relato de ter trabalhado na área da saúde antes de concluir o curso de Graduação em Enfermagem. Destes, a maioria dos trabalhadores $(65,8 \%)$ relatou serem auxiliares ou técnicos de enfermagem (dados não mostrados na tabela).

As características ocupacionais descritas (tabela 2) mostraram que um terço era contratado e proporção semelhante tinha função gratificada, ambas variáveis sem diferenças por sexo. Entre os homens a frequência de trabalhadores de plantões noturnos e com mais de um emprego foi mais elevada. A carga semanal média de trabalho profissional foi 55,1 $( \pm 20,9)$ e 61,4 horas $( \pm 21,8)$, respectivamente, para homens e mulheres $(p<0,001)$. Cerca de um terço dos trabalhadores referiram trabalhar em cargas superiores a 60 horas semanais, sendo que frequências mais elevadas foram observadas entre os homens (Tabela 2).

Tabela 2 - Características do trabalho profissional e doméstico dos enfermeiros segundo o sexo. Hospitais públicos do Município do Rio de Janeiro/RJ, 2010-2011.

\begin{tabular}{|c|c|c|c|c|c|c|c|}
\hline \multirow[t]{2}{*}{ Características } & \multicolumn{2}{|c|}{$\begin{array}{l}\text { Mulheres } \\
(n=2818)\end{array}$} & \multicolumn{2}{|c|}{$\begin{array}{l}\text { Homens } \\
(n=411)\end{array}$} & \multicolumn{2}{|c|}{$\begin{array}{c}\text { Total } \\
(n=3229)\end{array}$} & \multirow[t]{2}{*}{ Valor de $p$} \\
\hline & $\mathrm{n}$ & $\%$ & $\mathrm{~N}$ & $\%$ & $\mathrm{~N}$ & $\%$ & \\
\hline \multicolumn{8}{|c|}{ Trabalhava na área da saúde antes de ser enfermeiro } \\
\hline Sim & 657 & 23,5 & 125 & 31,3 & 782 & 24,5 & $<0,001$ \\
\hline Não & 2133 & 76,5 & 274 & 68,7 & 2407 & 75,5 & \\
\hline \multicolumn{8}{|l|}{ Servidor Público } \\
\hline Sim & 1866 & 68,1 & 259 & 64,8 & 2125 & 67,6 & $0,187^{*}$ \\
\hline Não & 876 & 31,9 & 141 & 35,3 & 1017 & 32,4 & \\
\hline \multicolumn{8}{|c|}{ Trabalha em plantões noturnos } \\
\hline Sim & 1672 & 59,3 & 307 & 74,7 & 1979 & 61,3 & $<0,001^{*}$ \\
\hline Não & 1146 & 40,7 & 104 & 25,3 & 1250 & 38,7 & \\
\hline \multicolumn{8}{|l|}{ Exerce função gratificada } \\
\hline Sim & 863 & 31,4 & 128 & 32,1 & 991 & 31,5 & $0,881^{*}$ \\
\hline Não & 1887 & 68,6 & 271 & 67,9 & 2158 & 68,5 & \\
\hline \multicolumn{8}{|l|}{ Número de vínculos } \\
\hline Um & 980 & 34,8 & 86 & 20,9 & 1066 & 33,0 & $<0,001^{* *}$ \\
\hline Dois & 1519 & 53,9 & 232 & 56,4 & 1751 & 54,2 & \\
\hline Três ou mais & 319 & 11,3 & 93 & 22,6 & 412 & 12,8 & \\
\hline \multicolumn{8}{|l|}{ Carga semanal de trabalho } \\
\hline Até 30 horas & 329 & 12,1 & 24 & 6,2 & 353 & 11,4 & $<0,001$ \\
\hline De 30 a 60 horas & 1478 & 54,4 & 191 & 49,2 & 1669 & 53,8 & \\
\hline Mais de 60 horas & 909 & 33,5 & 173 & 44,6 & 1082 & 34,9 & \\
\hline \multicolumn{8}{|c|}{ Supervisiona pessoas no trabalho } \\
\hline Sim, até 20 pessoas & 2034 & 73,0 & 276 & 68,3 & 2310 & 72,4 & $0,023 *$ \\
\hline Sim, 20 pessoas ou mais & 659 & 24,3 & 115 & 28,5 & 774 & 24,3 & \\
\hline Não & 94 & 3,4 & 13 & 3,2 & 107 & 3,3 & \\
\hline \multicolumn{8}{|c|}{ Pensou em abandonar a profissão (12 m) } \\
\hline Nenhuma vez & 1382 & 49,4 & 207 & 50,7 & 1589 & 49,6 & $0,171^{*}$ \\
\hline Algumas vezes & 1025 & 36,6 & 133 & 32,6 & 1158 & 36,1 & \\
\hline Frequentemente & 391 & 14,0 & 68 & 16,7 & 459 & 14,3 & \\
\hline \multicolumn{8}{|l|}{ Satisfação com a profissão } \\
\hline Satisfeito & 1474 & 52,8 & 230 & 56,4 & 1704 & 53,7 & $0,346 * *$ \\
\hline Indiferente & 680 & 24,3 & 88 & 21,6 & 768 & 23,7 & \\
\hline Insatisfeito & 640 & 22,9 & 90 & 22,1 & 730 & 22,6 & \\
\hline \multicolumn{8}{|c|}{ Procurou por emprego fora da enfermagem (nos últimos 30 dias) } \\
\hline Sim & 263 & 9,4 & 63 & 15,4 & 326 & 10,1 & $<0,001 *$ \\
\hline Não & 2537 & 90,6 & 347 & 84,6 & 2884 & 89,9 & \\
\hline \multicolumn{8}{|c|}{ Procurou por emprego na enfermagem (nos últimos 30 dias) } \\
\hline Sim & 869 & 31,1 & 111 & 27,1 & 980 & 30,6 & $0,107^{*}$ \\
\hline Não & 1928 & 68,9 & 298 & 72,9 & 2226 & 69,4 & \\
\hline
\end{tabular}

*teste de qui-quadrado de Pearson; ** teste de tendência linear $\quad$ excluiu-se ignorados. 
A supervisão é atividade frequente entre os enfermeiros estudados, sendo que entre os homens a coordenação de equipes numerosas foi um pouco mais elevada do que entre as mulheres. Cerca de metade dos trabalhadores pensou em abandonar a profissão nos últimos doze meses e quase um quarto se considera insatisfeita com a profissão, sem diferença entre os sexos. Cerca de 10\% dos trabalhadores esteve procurando emprego fora da Enfermagem nos últimos trinta dias, sendo que entre os homens essa frequência foi um pouco mais elevada. Cerca de $30 \%$ buscou emprego na própria enfermagem, sem diferença entre os sexos (tabela 2).

\section{DISCUSSÃO}

De forma geral, o estudo obteve alta adesão tanto pelos enfermeiros quanto pelos hospitais do município do Rio de Janeiro, em que se situa a maior rede hospitalar pública brasileira. As diferenças na adesão podem refletir diversos fatores relacionados à organização do processo de trabalho das equipes, que favoreciam e estimulavam, ou não, a participação dos enfermeiros.

Nesses hospitais, características importantes se diferenciaram entre os enfermeiros e as enfermeiras do estudo. Outros estudos nacionais ${ }^{(5-6,8)}$ e internacionais ${ }^{(11)}$ descreveram um perfil sociodemográfico semelhante em relação ao sexo, à idade e à situação conjugal: predominância feminina, casados e com idade média em torno dos 40 anos. No entanto, possivelmente por envolver apenas enfermeiros, e não os outros membros da equipe de enfermagem, observou-se frequências mais elevadas de trabalhadores que se auto referiram brancos e com renda per capita mais elevada quando comparamos nossos resultados a outros estudos com equipes de enfermagem ${ }^{(6,8)}$.

Embora uma expressiva parcela de enfermeiros tenha titulação relacionada a cursos de pós-graduação lato sensu, a proporção identificada é mais baixa do que aquela encontrada entre trabalhadores da atenção básica ${ }^{(11)}$. As formações relacionadas aos cursos stricto sensu (especialmente aos cursos de doutorado) têm baixa frequência e estão concentradas nos hospitais universitários e nos institutos federais (onde também é menor quando comparada ao hospital universitário de São Paulo) ${ }^{(12)}$. Uma das hipóteses que talvez explique essa baixa frequência pode estar relacionada aos critérios de inclusão do presente estudo - enfermeiros da assistência de enfermagem. A escassez de enfermeiros mais qualificados talvez se deva à exclusão dos ocupantes dos cargos de gestão, que tendem a apresentar titulação mais elevada. Acredita-se que tal escassez acabe por limitar o estímulo e a liberação de novos profissionais para os cursos stricto sensu que exigem maior tempo e dedicação para a formação. Outro aspecto pode estar relacionado ao tipo de vínculo dos enfermeiros nos hospitais, uma vez que cerca de um terço tinham contrato temporário. Além disso, o multiemprego, muito frequente entre os enfermeiros do estudo, talvez tenha influenciado na dificuldade em organizar uma agenda de dedicação, ou estabelecer como prioridade a formação profissional. Por fim, os critérios mais rígidos de seleção dos cursos de pós-graduação e a demanda reprimida de vagas podem representar fatores a considerar nos estudos que se dediquem ao aprofundamento desse achado.

Estes aspectos podem contribuir para a discussão do estabelecimento das prioridades no reforço à liderança científica e tecnológica da Enfermagem como ciência, o que ainda se constitui um desafio para a profissão. Esse será superado a partir do reconhecimento dos limites e entraves diagnosticados e por meio de esforços, buscando metas e produtos tecnologicamente mais avançados e com maior agregação de valor, especialmente do ponto de vista científico ${ }^{(17)}$. Portanto, estratégias que promovam a formação cientifica dos enfermeiros da assistência se fazem necessários.

Em relação ao processo de trabalho da Enfermagem, trata-se de uma organização pautada pela divisão técnica, social e relacionada ao gênero dos profissionais, hierarquização da equipe em categorias e predominância do sexo feminino(14). Embora constituam menor parcela da força de trabalho na Enfermagem, os homens relataram trabalhar mais à noite, terem múltiplos empregos na enfermagem, referiram carga de trabalho mais alta e supervisionarem equipes maiores. No entanto, conforme demonstrado em outros estudos nacionais ${ }^{(6,15)}$ a jornada doméstica dos homens é menor, quando comparados às mulheres da Enfermagem. Poucos estudos abrangentes aprofundaram o impacto dessas diferenças na saúde dos profissionais de enfermagem e no processo de cuidado no Brasil. Um estudo com população semelhante à do presente $\operatorname{artigo}^{(15)}$ demonstrou extensas cargas de trabalho (incluindo o trabalho doméstico) associadas a diversos comportamentos inadequados de saúde relacionados às doenças cardiovasculares entre as mulheres. Entre os homens o estudo também demonstrou alguns impactos da jornada profissional, especialmente em relação à inatividade física ${ }^{(15)}$.

Outro aspecto apontado nos resultados diz respeito ao acúmulo de horas semanais trabalhadas, relacionado ao multiemprego. A carga semanal média aqui identificada entre homens e mulheres foi superior àquela apresentada em outro estudo nacional realizado em São Paulo $(49,7)^{(6)}$, embora este tenha investigado todas as categorias profissionais de enfermagem. Foi também muito mais elevada do que aquelas observadas entre enfermeiros de diversos países europeus (que variou entre 24,8 na Holanda e 38,5 na Polônia) ${ }^{(16)}$. Embora não haja um limite seguro estabelecido para a duração das jornadas e cargas de trabalho, devido à variedade de condições envolvidas $^{(6)}$, é reconhecido que as cargas elevadas tanto afetam a saúde dos trabalhadores ${ }^{(6,15)}$, quanto a qualidade da assistência prestada ${ }^{(9)}$. Para exemplificar, um estudo da Associação Americana de Enfermeiros (ANA) demonstrou que o risco de erros aumentou significativamente quando a jornada diária era de 12 horas ou mais, quando se cumpriam horas extras, ou quando a carga ultrapassava 40 horas semanais ${ }^{(17)}$. Outro estudo, envolvendo hospitais dos Estados Unidos, encontrou uma jornada semanal média de 35 horas entre os enfermeiros e concluiu que, além de carência de pessoal disponível para o cuidado, a carga excessiva de trabalho dos enfermeiros está associada à mortalidade dos pacientes ${ }^{(18)}$.

Ancorados em valores professados pela categoria profissional desde a formação acadêmica, os múltiplos vínculos empregatícios são encarados como questão de sobrevivência, 
particularmente entre aqueles que trabalham sob o regime de plantão em hospitais. Os riscos para a saúde relacionados às longas jornadas, a ocorrência de acidentes e a possibilidade de ocorrência de erros no exercício profissional são usualmente minimizados e a adaptação às jornadas extensas parece ser tida como necessária ${ }^{(6)}$. Os baixos salários e a necessidade de complementação de renda justificam em parte a banalização das crenças atreladas aos riscos. Estudos que aprofundem análises acerca dos fatores determinantes das longas jornadas e respectivas consequências sobre o processo de cuidado precisam ser estimulados a fim de subsidiar estratégias de enfrentamento da situação no país.

$\mathrm{O}$ acelerado envelhecimento populacional, realidade também do Brasil, torna ainda mais necessária a profissão do enfermeiro. No entanto, nossos resultados já apontam para o desinteresse pela profissão, identificados por relatos de insatisfação e intenção de abandonar a carreira. Isso se expressa tanto em relação a pensamentos expressos quanto à efetiva procura por empregos fora da carreira. A intenção de abandonar a enfermagem, considerada um indicador que antecipa a efetiva saída, tem sido amplamente estudada nos países europeus $^{(19-20)}$ que descrevem a escassez de profissionais como um problema mundial(19). Ainda que o abandono precoce não seja um problema reconhecido no Brasil, vale ressaltar que a proporção de enfermeiros insatisfeitos e com intenção de abandonar a profissão, foi mais alta do que as apontadas pela maioria dos países europeus do Nurse's Early Exit Study (NEXT Study) ${ }^{(20)}$. É possível que as condições de trabalho adversas na maioria dos hospitais públicos cariocas expliquem em parte essas diferenças. Estudos que monitorem no tempo essas variáveis na população de enfermeiros brasileiros se fazem necessários, buscando antecipar e prevenir um fenômeno amplamente debatido nos países de renda mais alta.

Apesar da abrangência do presente estudo, algumas limitações podem ser apontadas. Dentre elas, as diferenças na adesão ao estudo nos diversos hospitais podem ter influenciado padrões mais próximos à realidade dos hospitais com maior numero de enfermeiros e naqueles em que a adesão foi mais alta. Essa adesão também poderia estar relacionada a algum grau de viés de autoseleção, uma vez que quem adere aos estudos podem ter características diferenciadas. Por fim, pode-se também esperar vieses de classificação, em função do padrão de respostas, aspecto que pode ocorrer em especial em estudos que utilizam de questionários autopreenchíveis em geral. Buscou-se minimizar esses aspectos utilizando instrumento pré-testado e perguntas e escalas já validadas em outros estudos, além de aplicar questionários com diagramação específica para autopreenchimento.

\section{CONCLUSÃO}

O presente artigo apontou aspectos desafiadores para a profissão de enfermeiros que atuam em hospitais públicos em um grande centro urbano brasileiro. Dentre estes, destacamos a baixa frequência de formação em cursos de mestrado e doutorado, além de cargas semanais extensas e os múltiplos vínculos empregatícios. A insatisfação e a intenção de abandonar a profissão já é mais frequente do que aquela observada em estudos internacionais. Em função de sua abrangência pioneira no País, os resultados do presente estudo podem subsidiar estratégias de melhorias das condições de trabalho dos enfermeiros nos cenários hospitalares.

\section{REFERÊNCIAS}

1. Merhy EE. O trabalho em saúde: olhando e experienciando o SUS no cotidiano. 3. ed. São Paulo: Hucitec; 2006.

2. Marques PA, Melo ECP. O processo de trabalho em uma unidade de terapia intensiva neonatal. Rev Esc Enferm USP 2011;45(2):374-80.

3. Costa RA, Shimizu HE. Atividades desenvolvidas pelos enfermeiros nas unidades de internação de um hospital-escola. Rev Latino-Am Enferm 2005;13(5):654-62.

4. Camelo SHH. Competência profissional do enfermeiro para atuar em unidades de terapia intensiva: uma revisão integrativa. Rev Latino-Am Enferm 2012;20(1):192-200.

5. Magnago TSBS, Lisboa MT, Griep RG, Kirchhof AL, Azevedo LG. Psychosocial aspects of work and musculoskeletal disorders in nursing workers. Rev Latino-Am Enferm 2010;18(3):429-35.

6. Silva AA, Rotenberg L, Fischer FM. Jornadas de trabalho na enfermagem: entre necessidades individuais e condições de trabalho. Rev Saúde Pública 2011;45(6):1117-26

7. Canini SRMS, Moraes SA, Gir E, Freitas ICM . Percutaneous injuries correlate in the nursing team of a Brazilian tertiary-care university hospital. Rev Latino-Am Enferm 2008;16(5):818-23.

8. Ferreira RC, Griep RH, Fonseca MJM, Rotenberg L. Abordagem multifatorial do absenteísmo por doença em trabalhadores de enfermagem. Rev Saúde Pública 2012;46(2):259-68.

9. Silva BM, Lima FRF, Farias FSAB, Campos ACS. Jornada de trabalho: fator que interfere na qualidade da assistência de enfermagem. Texto \& Contexto Enferm 2006;15(3):442-8.

10. Camerino D, Estryn-Behar M, Conway PM, Van Der Heijden BI, Hasselhorn HM. Work-related factors and violence among nursing staff in the European NEXT study: a longitudinal cohort study. Int J Nurs Stud 2008;45(1):35-50.

11. Ferrari RAP, Thomson Z, Melchior R. Estratégia da saúde da família: perfil dos médicos e enfermeiros, Londrina, Paraná. Semina Cienc Biol Saude 2005;26(2):101-8

12. Martins C, Kobayashi RM, Ayoub AC, Leite MMJ. Perfil dos enfermeiros e necessidades de desenvolvimento de competência profissional. Texto \& Contexto Enferm 2006;15(3):472-8. 
13. Erdmann AL. Formação de especialistas, mestres e doutores em enfermagem: avanços e perspectivas. Acta Paul Enferm 2009;22(Especial-Nefrologia):551-3.

14. Lopes MJM, Leal SMC. A feminização persistente na qualificação profissional da enfermagem brasileira. Cadernos Pagu 2005;(24):105-25.

15. Fernandes JC, Portela LF, Rotenberg L, Griep, RH. Jornada de trabalho e comportamentos de saúde entre enfermeiros de hospitais públicos. Latino-Am Enferm 2013.

16. Tackenberg $\mathrm{P}$, Hasselhorn HM, Büscher A. Nursing in Europe In: Working conditions and intent to leave the profession among nursing staff in Europe [acesso em $17 \mathrm{dez}$ 2012]:19-27. Disponível em: http://nile.lub.lu.se/arbarch/ saltsa/2003/wlr2003_07.pdf
17. Rogers AE, Hwang W-T, Scott LD, Aiken LH, Dinges DF. The Working hours of hospital staff nurses and patient safety. Health Aff 2004;23(4):202-12.

18. Trinkoff $A M$, Johantgen $M$, Storr $C L$, Gurses $A P$, Liang $Y$, Han K. Nurses' Work Schedule characteristics, nurse staffing, and patient mortality. Nur Res 2011;60(1):1-8.

19. Cortese CG. Predictors of critical care nurses' intention to leave the unit, the hospital, and the nursing profession. Open J Nurs 2012;(2):311-26.

20. Hasselhorn HM, Tackenberg $\mathrm{P}$, Müller $\mathrm{BH}$. Intent to leave nursing in the European nursing profession. In: Group Working conditions and intent to leave the profession among nursing staff in Europe [acesso em 17 dez 2012]:115-124. Disponível em http://nile.lub.lu.se/arbarch/saltsa/2003/wlr2003_07.pdf 